\title{
Dual Device User Interface Design for Ubiquitous Language Learning: Mobile Phone and Interactive Television (iTV)
}

\author{
Sanaz Fallahkhair \\ School of Computing \\ University of Brighton, UK \\ s.fallahkhair@brighton.ac.uk
}

\author{
Lyn Pemberton \\ School of Computing \\ University of Brighton, $U K$ \\ lyn.pemberton@brighton.ac.uk
}

\author{
Richard Griffiths \\ School of computing \\ University of Brighton, $U K$ \\ r.n.griffiths@brighton.ac.uk
}

\begin{abstract}
In this paper we describe the design and development of a system that facilitates language learning from a combination of two devices, interactive television (iTV) and mobile phone. We present a number of requirements for technologies to support informal language learning based on theories of language learning, theories of formal and informal learning, our own studies of adult language learners and the affordances of $i T V$ as a medium to support learning. We describe TAMALLE (Television and Mobile phone Assisted Language Learning Environment), a prototype system based on these requirements and discuss some of the user interface design issues that arise in the context of cross platform dual device systems for ubiquitous learning.
\end{abstract}

\section{Introduction}

The possibility of using "non-desktop" technologies for supporting learning opens up a wide variety of activities and interactions for learners. The potential for technologies such as mobile devices (m-learning) and interactive television (t-learning) for learning has provided a new direction for research in the field of educational technology. In particular, designing for these new technologies has thrown up a number of design challenges associated not only with the physical and functional limitations of these devices, but also with the effective implementation of new learning paradigms - situated, collaborative, life-long, personalized and contextual $[1,2,3]$.

"Beyond the desktop" technologies are currently being harnessed to support these learning paradigms. However each technology has distinct characteristics that facilitate some types of use and hamper others. For example, whilst mobile device afford a wide variety of personal activities and learning on move, they are less powerful for enabling learning from authentic and immersive content. In contrast, television provides rich multimedia presentation of authentic and immersive content that is constantly renewed. Programmes such as news, soap-operas and documentaries have the potential to enhance language learners' experience by showing the target language, culture and context of use. However, iTV does not naturally provide facilities for personalised learning or learning on the move in the way that mobile devices do. Designing in order to take advantage of what each device does best is the primary focus to the TAMALLE project.

Despite Robertson et al's pioneering CHI paper discussing coordinated iTV and PDA interaction [4], little is known about dual device interaction in general or about such interaction in the context of educational technology applications in particular. In this paper we concentrate on the design and development of the interfaces for a dual device system for informal language learning. The system aims to capitalise on the strengths of two specialised technologies, iTV and mobile phones, which tend to be used in different settings and at different times. We first briefly overview the existing literature on mobile and iTV technologies for language learning. We then discuss briefly the first stage of the project, learner-centred negotiation of requirements, for developing ubiquitous language learning, which led us to the design and development of the TAMALLE system, whose technical architecture is sketched. The interfaces for the two devices are then described.

\section{Mobile technologies for language learning}

The potential value of learning via mobile devices or m-learning has been widely realised [5, 6]. Mobile devices enhance learning experiences by enabling communications, learning on the move, and on an "anytime and anywhere" basis [7]. For language learning in particular this realisation also holds true. Second Language learners currently are often to be 
found with a pocket dictionary or a personal vocabulary book. As a result several researchers have begun to investigate the potential of mobile devices for language teaching $[8,9,10,11]$.

Godwin-Jones points out how mobile and wireless technologies could provide an opportunity for language and cultural learning. He describes a project to develop wireless system called RAFT that can be used on student's field trip. RAFT helps an individual to store and retrieve information regarding their field trip on their handheld mobile device and to share it with other learners. Although RAFT was not specifically designed for language learning, its developers suggest that it could be used for cultural and language learning by learners who are on a trip abroad, e.g. to conduct interviews with native speakers, and to share it with other learners [10].

The AD-HOC project aims to develop mobile language learning environment to facilitate 'learning on demand' for European travellers who want to acquire language skill in order to communicate with local people. The AD-HOC system acts as a tutor to teach linguistic and cultural knowledge through the use of multiple media presentations (e.g. text, sound, picture and video). The language learning environment offers representations of contextualised, authentic, real life situations for different level of competency and within different thematic fields (e.g. business travel, travelling of young people, etc.).The underpinning pedagogical principle of the AD-HOC project is self-directed learning [8].

The M-learn project is concerned to develop a mobile learning system for young adults (16-24) in order to teach some aspects of literacy and numeracy, and to involve them in the development of their lifelong learning [12].

The mobile language learning system, designed in Finland [9], delivers lessons using sound and text to teach grammar and vocabulary. It tracks the learner's progress and integrates voice technology for user interaction.

BBC Worldwide provides an English language teaching service via mobile phone in China. Learners receive a daily text message on their mobile containing a phrase in English together with the Chinese translation. A range of topics are covered (e.g. sport, business, lifestyle, etc.). The idea behind the system is to provide an opportunity for busy learners on the move to learn authentic spoken English [13].

A project conducted in Taiwan developed a mobilebased (PDA) interactive language learning environment for elementary school children learning English as a second language. The activities aimed to help students to learn listening, reading and writing skills. For example, a scenario to teach words related to images showing a body parts provides a word's pronunciations and spelling when the image is clicked by the user. Evaluation showed a positive response from learners and indicates that the use of mobile devices can significantly increase student motivation and interest [11].

The Speak My Speak project is investigating the use of SMS (Short Messaging Service) as a communication tool between adult English language learners and native English tutors. They conclude that using SMS in language learning is feasible and promising students did reflect on texts sent and received, and were active in constructing the content of communication [14].

MobiLearn software provides a mobile phrase book in different languages for pocket PC. The main aims of the software are to provide a list of common words and phrases, to enable learners to bookmark their required words and phrases for easy access, to hear pronunciations and to test their knowledge through a number of quizzes provided.

The INLET project (Lingua) developed an innovative mobile phone support system to encourage tourists to learn Greek language at the Athens Olympic Games 2004 [15]. The system provided a number of facilities for learning useful Greek phrases in a just-intime manner. Language categories judged most beneficial for tourists were developed as follows: "basic" (e.g. greeting, numbers, basic words), "where" (e.g. phrases for asking direction, going by bus, taxi and trains), "when" (e.g. asking times, today, now, tomorrow), "Olympic Sport" (games name, athletics, fencing, etc.) and "buying" (asking price, money, expressions like expensive, cheap, etc.). Users, recruited at the airport in many cases, were able to register for SMS messages to be sent to their mobile phones freely and regularly containing useful phrases. They also could request SMS translations of other languages into Greek.

\section{3. (I) TV for language learning}

ITV is a new media technology that has great promise for language learning [16, 17, 18]. Before turning to interactive $\mathrm{TV}$ it is worth considering television which itself is already a powerful learning environment for language learners. Television offers a rich multimedia experience, where learners can immerse themselves in authentic materials from the target language and culture. This material may well be engaging in itself, with up-to-date ever-changing content displaying a range of speakers and contexts. Many television shows constitute important cultural events in their own right providing a shared reference for people sharing or aspiring to share a culture. In its 
non-interactive state, it clearly affords watching, reading and listening, making it an excellent medium for learners to practice comprehension skills and also to acquire background cultural knowledge. Comprehension of spoken material is strongly supported. Sherrington [19], exploring the potential of conventional television for language teaching, notes that a number of listening skills can easily be practised via television, including recognising and understanding:

- Segmental and supra-segmental features

- Vocabulary items, short phrases and longer segments of speech

- Syntactic structures

- Varieties of speech, such as registers and dialects

- Discourse patterns

- Pragmatically determined features

Lonergan points out the benefits of viewing TV programmes in the target language, particularly TV's multimedia aspect: "the suitability of television as a medium for bringing a living language to learners is undoubted. The dynamic combination of sound and vision can bring an air of reality into the classroom. The wealth of visual information available can convey the atmosphere of another culture, can show paralinguistic aspect of communication; the techniques of television can present material to learners in ways quite beyond the resources of the language teacher" [20].

One disadvantage of TV broadcast over video or DVD is its "non-interruptible quality" where it restricts learners from replaying the information available [21, p.3]. Broady points out that one of the problems associated with target language $\mathrm{TV}$ is that the foreign language learners are not aware of the required background knowledge - something she refers to as "cultural knowledge" - that needs to be acquired in order to understand the programme. She discusses the notion that a situation can be misunderstood by nonFrench viewers when the accompanying image does not support interpretations of the "verbal input" in watching a French News channel. She further argues: 'because it [television] conveys 'real' language used by 'real' people in 'real' situation, it is generally perceived as motivating and interesting by learners. Yet this very cultural authenticity can render it frustratingly obscure. But is this a 'real' problem?". She latter mentions that "non-native viewers are not the only ones whose understanding is impaired when picture and commentary do not correspond closely: it happens to native viewers too." [21, p.4]. One problem that non-native speakers sometimes have is that they "often lack the confidence... and assume that they need to understand every word. With such strategy, the richness of authentic television are likely to remain buried." [21, p.5]. For this reason, a number of researchers attempt to develop "viewing strategies" that could be used by learners when watching authentic television. These strategies explain how to maximize comprehension of foreign TV viewing [22].

Several projects $[23,24,25]$ have analyzed the use of TV with first language subtitles (L1 subtitling) and second language subtitles (L2 subtitling), as an aid to comprehension, retention of second language vocabulary and improving reading skills. One such study suggests that even TV with an L2 audio track and L1 subtitling could lead to incidental second language learning [22]. Fridman argues for closed captioned videos as powerful tools for improving vocabulary and reading comprehension skills for EFL learners. Bean \& Wilson report the motivating influence of captioned television, and positive attitudes on the part of learners toward this medium [26]. Neuman and Koskinen suggest [27] that captioned television can be used as an effective instructional tool in learning vocabulary and concepts. Koskinen et al. studied the effect of captioned television on incidental vocabulary acquisition by adult ESL learners. They assessed vocabulary knowledge of viewers who watched TV with and without captions, identifying "a statistically significant difference in favour of captioned TV" and "a positive relationship between oral English language competency and vocabulary learning". The participants with higher levels of oral proficiency learned more than less proficient subjects [24, p.368].

Borras and Lafayette investigated the effect of L2 subtitles on comprehension and reading skills. They compared the performance of learners who had used video with and without subtitles. The result clearly favoured the subtitle option and they conclude that "when learning from 'authentic video' in a multimedia environment, having the opportunity to see and control subtitles, as opposed to not having that opportunity, result in both better comprehension and subsequent better use of the foreign language" [28, p.82].

Digital television adds a new dimension to learning from the TV by multiplying available channels [22, 29]. However, this is an increase in the quantity of available material rather than a change in the type of affordance provided by the medium. It is essentially more of the same. Digital interactive television (iTV) offers genuinely new ways of using the television set. Interactivity adds new facilities for information retrieval and communications [30]. With interactivity viewers could:

I. Select from alternative audio/ video streams 
II. Make their own choice amongst subtitling or captioning options

III. View supplementary information on screen to access before, during or after a broadcast

IV. Use communication tools such as chat /email.

The functionality provided by iTV is similar to that provided by the Internet, but it is displayed on the familiar TV screen. Despite the fact that current levels of interactivity are relatively limited, constrained by the components of the iTV set up, i.e. the set-top box and its software, the on-screen display and the remote control, the potential is clearly vast.

\section{Learners' requirements}

To inform the design of the iTV language learning system, we conducted three focus group studies to investigate the approaches that a number of independent adult language learners have adopted towards their language learning and their attitudes towards a range of technologies including iTV and mobile phones $[1,2,36]$. A set of general requirements emerged:

- Support informal learning rather than formal

- Support learning in context

- Support learning while immersing into the environment

- Support learning from engaging and authentic materials

- Support learning on move (anytime and anywhere basis)

- Support learners understanding by scaffolding

- Support learners in creating and managing their own personal knowledge and sphere

- Provide a support for just-in-time learning

- Support learning in an unobtrusive fashion (by not introducing a new device or imposing educational materials on fellow viewers)

\section{Prototype description}

The prototype of dual device language learning support system via iTV and mobile phones (TAMALLE) was designed based on the requirements outlined in Section 4. TAMALLE is an informal language learning environment that has dual interfaces across iTV and mobile phones and can support learning from authentic television programmes such as news, soap-opera, documentaries, etc. A dual language learning sphere on both iTV and mobile phone devices allows learners to incorporate edutainment with their language learning experiences. To this end, the system provides support for comprehension of specific language items for viewers as they watch a foreign language television programme that is pitched at a level slightly above their current level of language competence. These language items can be incorporated by learners into their learning sphere, which is also accessible via their mobile phone.

TAMALLE is also a context aware system, in that the mobile sphere supports learning in the context of the TV programme. The television provides authentic materials and a context for learning. The mobile can scaffold learners' understanding of the programme by enabling them to access the summary of programme as well as difficult language items, such as vocabulary, phrases, etc that may appear inside a programme. These language items can be accessed prior to, during and after the show. Learners are also enabled to add, search and remove these language items from/into their personal spheres. Even without television, the mobile is still useful as tool for learning a new language items and as a tool for managing personal knowledge.

Annotation based support is provided to scaffold difficult language items and culturally specific knowledge that helps learners in understanding the programme while watching. If more unknown terms were found, the TAMALLE dictionary could help to check meaning and giving examples. Section 5.1 discusses the technical architecture of TAMALLE and section 5.2 describes TAMALLE dual interfaces.

\subsection{Dual device architecture}

We have investigated two possible end-to-end solutions based on a multi-tier client/server architecture consisting of the broadcast-end tier, the back-end tier and front-end tier for developing the language learning service.

One solution is to develop a learning management system that can be located in the broadcast-end or back-end tier. This learning management system provides content to both set top box and mobile devices and also holds learning content or learning objects in a database on the back-end tier (MySQL). In the front-end tier we have the set top box and WAP enabled mobile devices. Two way communications can be established between set top box and back-end tier through telephone modem, ADSL or broadband cable, while mobile phone devices communicate with the back-end tier through the WAP protocol. For interactive SMS messaging, we can use SMS gateway providers; the one that we are using in UK is SMS2mail provider. This architecture is illustrated in Figure 1 and for TAMALLE development we used this architecture. 


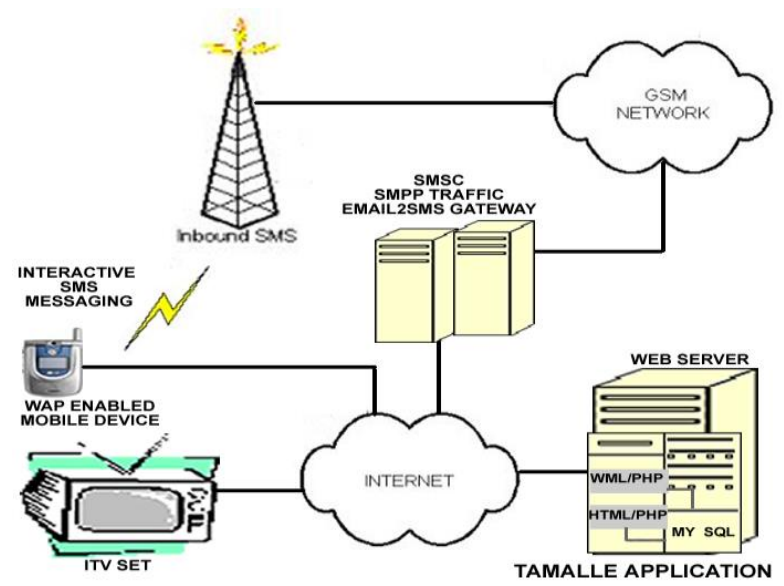

Figure 1: TAMALLE dual device architecture

An alternative solution is to use Digital Video Broadcasting (DVB), Java enterprise solution and Bluetooth [31]. The language learning content and mainstream television programme can be encoded and multiplexed before being broadcast via the DVB stream. The learning content will be retrieved by a client based Java application located in an MHP based set top box that also provides the API required for content retrieval and presentation to the mobile devices.

\subsection{Dual device interface}

Our design of the prototype interface had five aims:

I. to use the specialised device most suitable for the learning task in question;

II. to use the appropriate physical characteristics and learning affordances of each device both alone and in combination;

III. to support individuals as well as group of learners who may or may not be interested in learning;

IV. to allocate learning content to a device that will be most suitable for providing a particular mode of presentation;

$\mathrm{V}$. to provide consistency in terms of look (navigations, icons, words) and feel (learning tasks, activities and contents) across dual devices to ease the learnability of the overall system.

ITV was combined with mobile phones rather than other portable devices, such as laptops or PDA. Viewers are already using mobile device to interact with iTV applications, i.e. SMS voting, playing along a quiz show. Mobile phones could play the role of a companion device that has some specialised features [32], which may offer more personalised learning materials for people who are sharing the television.
Technically both iTV and the mobile are capable of displaying and manipulating learning materials, which in this case are television programmes and accompanying textual annotations or other information. However, each device is different in terms of their strength. It becomes clear that we need to study the capability of each device for supporting a learning task. For example, television is more appropriate for delivering picture, videos and audio materials, especially in combination. Mobile phones are suitable for displaying text and some graphics. Thus in our dual device scenario, iTV is preferred for taking care of presentation of videos, photos, spoken and textual materials where the mobile phones augment that with more textual information and annotations.

There are other issues concerning the physical characteristics and limitations of each device, such as screen size, resolution and memory capabilities, which constrain the user interactions possible. ITV users are limited to a menu-style interface with navigation and action carried out via the remote control or in some cases with an infrared keyboard. The remote control offers interaction via coloured key (red, green, yellow and blue); numbered keys (0-9), arrow keys (up/down, right/left) and an OK action button. The screen interface is similarly constrained. We followed guidelines developed by the $\mathrm{BBC}$ to decide about factors such as font style, size, contrast and positioning $[33,30]$.

Interaction styles with mobile phones are also limited in various ways: small screens (i.e. amount of data that can be displayed on one screen, as well as the size and placement of graphical, textual elements and navigations), soft key use (soft keys for selection and navigations are different in many phones) and memory constraints [37]. In designing TAMALLE, these constraints are tackled mainly by simplifying navigation, making navigation controls very salient and minimising the navigation depth. This is in keeping with the "simple and shallow" notion that requires minimizing the number of screens while keeping an appropriate amount of scrolling, balancing breadth and depth, therefore improving the usability and learnability of overall system [34].

In the following section we describe the main functionalities of TAMALLE and show how these are displayed and used on iTV and mobile phones. Four types of functionality are described: 1) scaffolding difficult language items, 2) scaffolding overall understanding, 3) just-in-time scaffolding and 4) managing personal learning sphere.

The TAMALLE application on the iTV side is activated by using the conventional "call to action", i.e. pressing the red button on the remote control, while watching a programme. From this point the viewer sees 
the news streamed into the TAMALLE application. Login is offered but not obligatory for nonpersonalised services. The broadcast programme appears reduced on the right side of the screen with interactivity on the left. For mobile use, learners are required to use a WAP enabled mobile phones to connect to the TAMALLE mobile application. Both interfaces are illustrated in Figure 2.
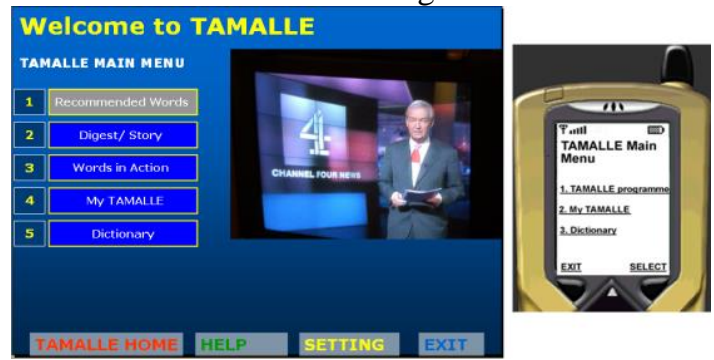

Figure 2: TAMALLE main menu

5.2.1. Just-in-time scaffolding. The system provides just-in-time help for difficult cultural or language items as they appear in the programme. By pressing "Words in Action" from the TAMALLE main menu the just-intime support will be activated providing textual annotation similar to subtitles on the television screen. The individual items may explicate a word (e.g. Tory = Conservative) or identify a scene or individual (This is 10 Downing St - the Prime Minister's residence). The reason that our design locates the call-to-action dialogue on the iTV side rather than the phone is due to the fact that this just-in-time scaffolding will be only beneficial during the programme show time and not before and after. However, a mobile can augment justin-time support while watching with other fellow viewers who may not be interested in learning a language. The learner may not want to impose annotations on everyone in the room. In this case, they can send a text message to service whose number is displayed briefly on the television screen to get just-intime scaffolding on their mobile phone (see Figure 3).
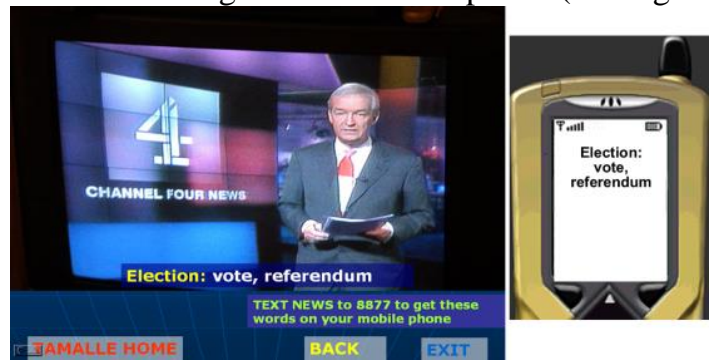

Figure 3: Just-in-time scaffolding

5.2.2. Scaffolding difficult language items. Difficult or unusual language items from the dialogue or commentary will be transcribed for TAMALLE viewers. Viewers who are logged in may select
"Recommended Words" to see a list of language items with explanation that can also be added to their personal learning sphere (My TAMALLE), which is also accessible via mobile phone (see Figure 4). The main interaction with the TAMALLE application on the iTV side is by remote control, with the red key taking them to the home page, the yellow key leading to the previous page, and the blue key to exit the application. The arrow keys move the selection up and down the list, while the Select key allows adding a chosen word to a learner's personal sphere. On the mobile interface, a selected word is highlighted and could be added by pressing the handset's select key.

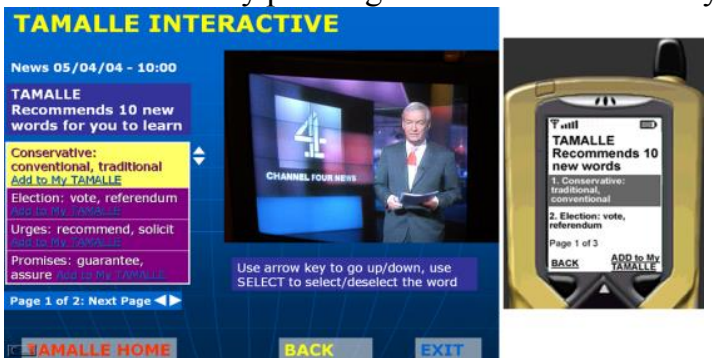

Figure 4: Scaffolding difficult language item

5.2.3. Scaffolding overall understanding. The viewer's overall understanding will be improved by providing a summary of programme content. This will differ according to genre, with the news being summarised as headlines, a drama as a brief plot summary and so on. This is accessed via "Summary/Digest" on the main menu.

This is augmented by an on-screen dictionary. In the following screenshot, a news digest is provided on the left hand side of TAMALLE iTV application, activated by the green button on the remote control. The mobile phone version also provides a link to a programme summary that can be accessible before, during or after the show and on move. Again this is augmented by a dictionary.

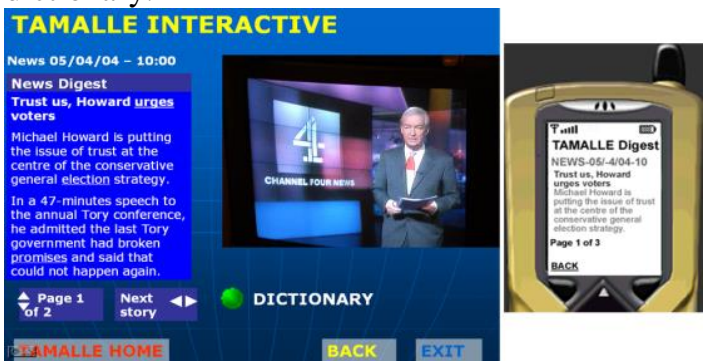

Figure 5: Scaffolding overall understanding

5.2.4. Managing personal learning sphere. The system enables learners to manage their personal "learning sphere," accessible via iTV and mobile interface. The recommended words can be added to a personal vocabulary list for later practice. Learners can 
view all their saved language items from the main menu. They can also search for specific language items and remove those no longer wanted. Figure 6 shows "My TAMALLE" on both iTV and mobile device.
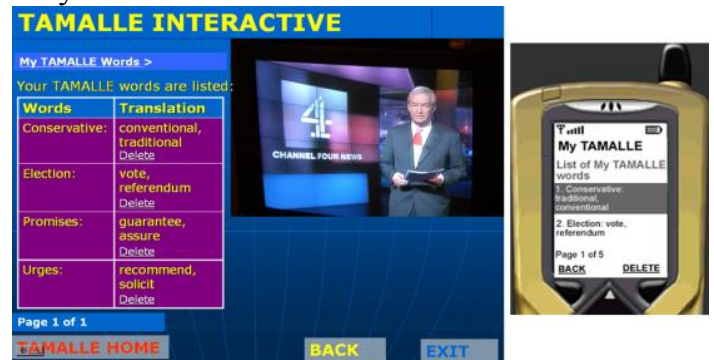

Figure 6: Managing personal learning sphere

\section{Discussion}

In this section we explain some of the reasoning behind the design decisions. Simplicity and consistency were our ultimate criteria in designing TAMALLE. Simplicity is imposed by the constraints of the two devices, while consistency, both internal and external, will make for ease of use and learnability. To this end, the mobile phone version of the system can follow the conventions of the phone on which it is viewed.

This design solution is not the only one possible: another team could start with similar requirements and ends up with quite a different set of choices for functionality and interaction design. The chosen design was very much influenced by the research of AlSeghayer [35] who demonstrates significant improvements in language comprehension and incidental learning where a combination of media audio, annotated text and video - were used.

For navigation and function activation, the iTV system has been designed to make user interactions as simple as possible using appropriate coloured and numbered keys of the remote control. Each menu item also has a numerical label allocated to it, giving an alternative selection mechanism. The navigation throughout the system is also consistent using coloured controls at the bottom of each page: Home (red), Back (yellow), Exit (blue). From the mobile phone interface the learners can move up and down the list of menu options using the direction keys and use the right and left soft key to choose a required option. The back button in mobile interface is consistent throughout the pages and always takes the learner to the previous page.

Media choice may also be worth commenting on. The justification for providing text annotations for justin-time support was research by Koskinen et al. showing that combined video and textual annotation of spoken language in a from of subtitle or closed caption could aid learning vocabulary, improving listening, comprehension and reading skills [27, 24].

The decision of whether to display video on the mobile phone, on the other hand, was based on our understanding of the affordances of the device. Despite the fact that, with the advent of the DVB-H standard, television can also be viewed on mobile phone screens, the physical limitations make this a much less attractive option for providing all the TAMALLE functionalities required for language learning. Television clearly affords watching more than the mobile phone. People already have a very well established relationship with their television set, which we have chosen not to disrupt. Television is the device of choice for viewing broadcast video, while the mobile phone, on the other plays the role of a companion device that is also functional as a standalone to support learning on the move.

\section{Conclusions and further work}

The TAMALLE design responds to the requirements we derived from multiple sources. Learning from engaging, up-to-date and authentic materials that are of intrinsic interest to language learners is enabled. Learning in context is made possible, with rich multimedia content providing a comprehensible setting for the new language. Learning on the move is supported, while the leisure use of television is respected. Learners can also choose to take advantage of one device without the other. The scaffolding learning opportunities can aid in acquiring lexicon items and to improve learner's comprehension and listening skills. The textual annotations can facilitate just-in-time support for learning cultural specific knowledge and difficult language items. Finally, TAMALLE supports learners in creating and managing their own personal language knowledge accessible in anytime and anywhere basis.

However, the design of TAMALLE as a dual device learning service raises a number of questions to be addressed in further research. A first question regards the source and nature of the support material. Ideally we would have liked to find guidance in the language teaching literature on selecting individual words or phrases for attention. However, little practical guidance is available to help us make a reasoned choice. Without such rules or guidelines, automating the functionality of TAMALLE, which would be necessary if it is to be widely used and sustainable, will not be possible. In addition, it may well be useful to tailor this support material to the learner's level of competence, motivation, experience and so on. We are currently 
conducting experiments with language learners to try to derive guidelines in this area.

A second set of issue is concern with the methodology to evaluate the dual interface devices such as TAMALLE and its implications for learning. At the moment we are considering evaluating the system in terms of its usability but will need to develop new techniques tailored to the dual device scenario.

\section{References}

[1] Pemberton, L., Fallahkhair, S. \& Masthoff, J. (2004). Towards a Theoretical Framework for Informal Language Learning via Interactive Television. Proceeding of CELDA 2004 Portugal. pp.27-34

[2] Fallahkhair, S., Masthoff, J. \& Pemberton, L. (2004a). Learning Languages from Interactive Television: Language Learners Reflect on Techniques and Technologies. Proceeding of EdMedia 2004 Switzerland. pp.4336-4343 [3] Naismith, L., Lonsdale, P., Vavoula, G. \& Sharples, M. (2005). Literature review in mobile technologies and learning. Nesta Futurelab.

[4] Robertson, S., Wharton, C., Ashworth, C. \& Franske, M., Dual Device User Interface Design: PDA and interactive Television, Proceedings of CHI 1996 Vancouver. [5] Sharples, M. (2000). The design of personal mobile technologies for lifelong learning. Computers and Education, 34, pp.177-193

[6] Leung, C. \& Chan, Y. (2003). Mobile Learning: A New Paradigm in Electronic Learning. Proceeding of $3^{\text {rd }}$ IEEE International Conference on Advanced Learning Technology. [7]Roschelle, J. (2003). Keynote paper: Unlocking the learning value of wireless mobile devices. Journal of Computer Assisted Learning, 19, pp. 260-272.

[8] Malliou, E., Miliarakis, A., Stavros, S., Sotiriou, S.A., Startakis, M., \& Tsolakidis, C. (2002). Proceedings of the European Workshop on Mobile and Contextual Learning. [9] Kadyte, V. (2003). Learning can happen anywhere: a mobile system for language learning. LSDA publication. [10] Godwin-Jones, B. (2004). Emerging Technologies Language in Action: From Webquest to virtual Realities. Language Learning and Technology. 8(3).

[11] Tan, T. \& Liu, T. (2004). The mobile interactive learning environment (MOBILE) and a case study for assisting elementary school English learning. Proceeding of ICALT 2004 Finland. pp.530-534.

[12] Ultralab. (2003). M-learn project. http://www.ultralab.ac.uk/

[13] BBC World Wide Press Release. (2003). BBC to provide English language teaching in China via mobile telephones,http://www.bbc.co.uk/

[14] Markett, C. (2003). Speak my speak: A feasibility study of the use of mobile phone text messaging with learners of English as a second language. http://www.cs.tcd.ie / [15] Pincas, A. (2004). Using Mobile Support for Use of Greek During the Olympic Games 2004. Proceeding of Mlearn conference 2004.

[16] Atwere, D. and Bates, P., 2003. Interactive TV: a learning platform with potential. LSDA Publication.
[17] Pemberton, L., 2002. The Potential of Interactive Television for Delivering Individualised Language Learning, Proceeding of ITS conference San Sebastian.

[18] Underwood, J., 2002. Language Learning and Interactive TV. Proceeding of ITS conference San Sebastian. [19] Sherrington, R. (1973). Television and Language Skills. Oxford: Oxford U.P.

[20] McGovern, J. (1983). Video Applications in English Language Teaching, ELT. Oxford: Pergamon.

[21] Broady, E. (1997). Old technology, new technology: Video makes a come-back. In A. Korsvold \& B. Ruschoff (eds). New technologies in language learning and teaching. [22] Meinhof, U. (1998). Language Learning in the Age of Satellite Television. Oxford U.P.

[23] Bean, R.M. \& Wilson, R.M. (1989). Using closed captioned television to teach reading to adults. Reading Research and Instruction, 28(4), pp. 27-77.

[24] Koskinen, P., Knable, J., Markham, P., and Jensema, C. \& W.Kane, K. (1996). Captioned television and the vocabulary acquisition of adult second language correlational facility residents. Journal of Educational Technology Systems, 24(4), pp. 359-373.

[25] Koolstra, C.M. \& Beentjes, J.W.J. (1999). Children's vocabulary acquisition in a foreign language through watching subtitled television programs at home. Educational Technology Research and Development. 47(1), pp. 51-60. [26] Friedman, A. R. (2001). Using Closed Captioned Video in the ESL Reading Class. http://www.umkc.edu/cad/nade/ [27] Neuman, S., \& Koskinen, P. (1992). Captioned television as comprehensible input: Effects of incidental word learning from context for language minority students. Reading Research Quarterly, 27(1), pp.95-106.

[28] Chapelle, C.A. (2003). English Language Learning and Technology. Amsterdam/Philadelphia: John Benjamins B.V. [29] Moores, S. (1996). Satellite television and everyday life. Luton: Luton University Press.

[30] Gawlinksi, M. (2003). Interactive television production. London: Focal Press.

[31] Fallahkhair, S. (2004). Media Convergence: An architecture for iTV and mobile phone based interactive language learning. Proceedings of EuroITV Brighton. [32] Cereijo Roibas, A. and Sala, R. (2005). Beyond the remote control: Going the extra mile to enhance iTV access via mobile device $\&$ humanizing navigation experience for those with special needs. Proceedings of EuroITV Denmark. [33] BBC, (2002). Interactive Television Style Guide. http://www.bbc.co.uk/commissioning/styleguide2_1.pdf [34] Kiili, K., (2002). Evaluating WAP Usability: "What Usability?". Proceedings of WMTE 2002.

[35] Al-Seghayer, K., (2001). The effect of multimedia annotation modes on L2 vocabulary acquisition. Language Learning \& Technology, 5(1), 202-232.

[36] Fallahkhair, S., Pemberton, L. \& Masthoff, J. (2004b). A dual device scenario for informal language learning: interactive television meets the mobile phone. Proceeding of the $4^{\text {th }}$ IEEE International Conference on Advanced Learning Technologies, pp.16-20.

[37] Passani, L. Building Usable Wireless Application for Mobile Phones.(2002). Proceeding of Human Computer Interaction with Mobile Devices. pp. 9-20. 